\title{
PALHA DE VARIEDADES DE SORGO NA FORMULAÇÃO DE NOVOS COMPOSTOS PARA O CULTIVO DO COGUMELO Pleurotus ostreatus
}

\author{
Otavio Augusto Pessotto Alves Siqueira* \\ Olívia Gomes Martins** \\ Meire Cristina Nogueira de Andrade***
}

RESUMO: Pleurotus ostreatus é um cogumelo comestível cujo cultivo pode ser realizado em diferentes resíduos como substrato, porém pouco se sabe sobre a utilização do sorgo para sua produção. Objetivou-se neste estudo analisar a viabilidade do sorgo para o cultivo de $P$. ostreatus. Foram testados três tratamentos, C1 (testemunha, palha de cana-de-açúcar), C2 (sorgo biomassa) e C3 (sorgo sacarino). Os tratamentos foram submetidos a processo de compostagem e pasteurização e após o resfriamento dos compostos foi realizada a inoculação de $P$. ostreatus. A incubação e colheita foram realizadas durante 2 meses, seguidas de análise nutricional dos cogumelos e análise química dos compostos. Foram avaliados eficiência biológica, perda de matéria orgânica e massa de basidiomas frescos. Não houve diferença significativa entre os tratamentos, segundo análise química dos substratos, exceto para o teor de umidade, em que o tratamento testemunha foi inferior aos demais. Quanto à produtividade, os tratamentos apresentaram os seguintes resultados: $\mathrm{C} 1: 1,73 \mathrm{~kg}$; $\mathrm{C}$ : 1,34 kg; e C3: 1,07 kg. Não houve diferença significativa para a eficiência biológica entre os tratamentos $\mathrm{C} 1$ e $\mathrm{C} 2$, com valores de $69,80 \%$ e 72,76\% respectivamente, enquanto o tratamento C3 apresentou a menor eficiência biológica (51,72\%). A perda de matéria orgânica foi de 50,56\%, para C1, 44,50\% para C2 e 40,16\% para C3. A análise nutricional dos cogumelos não demonstrou diferença significativa entre os tratamentos, exceto para o teor de fibra bruta, onde o tratamento $\mathrm{C} 3$ foi superior com $18,95 \%$, seguido pelo tratamento C2 com $16,40 \%$ e o testemunha com $14,95 \%$.

PALAVRAS-CHAVE: Análise nutricional; Fungos; Produtividade; Resíduos.

\footnotetext{
" Mestrando em Agronomia (Agricultura) pela Faculdade de Ciências Agronômicas, Universidade Estadual Paulista (UNESP), Botucatu, Brasil.

** Mestranda em Agronomia (Energia na Agricultura) pela Faculdade de Ciências Agronômicas, Universidade Estadual Paulista (UNESP), Botucatu, Brasil.

*** Doutora em Agronomia, docente permanente do Programa de Pós-graduação em Agronomia (Energia na Agricultura), Faculdade de Ciências Agronômicas, Universidade Estadual Paulista (UNESP) Botucatu, Brasil. E-mail: mcnandrade@hotmail.com
} 


\title{
STRAW FROM DIFFERENT SORGHUM VARIETIES IN THE FOR- MULATION OF NEW COMPOUNDS FOR THE CULTIVATION OF Pleurotus ostreatus
}

\begin{abstract}
The cultivation of the edible mushroom Pleurotus ostreatus may be performed in different substrates. No data is available on the use of sorghum wastes for mushroom production. Current study evaluates the feasibility of sorghum in the cultivation of $P$. ostreatus. Three treatments were tested: C1 (control, sugar cane straw), C2 (sorghum biomass) and C3 (saccharin sorghum). Treatments were composted, pasteurized and, after cooling, $P$. ostreatus was inoculated. Incubation and harvest occurred during two months, followed by nutrition analysis of the mushrooms and the composts' chemical analysis. Biological efficiency, loss of organic matter and mass of fresh mushrooms were evaluated. According to the substrates' chemical analysis, there was no significant difference among treatments, except humidity, where control was lower than the others. Treatments had the following productivity: C1: $1.73 \mathrm{~kg}$; C2: $1.34 \mathrm{~kg}$; $\mathrm{C} 3: 1.07 \mathrm{~kg}$. There was no significant difference for biological efficiency between treatments $\mathrm{C} 1$ and $\mathrm{C} 2$, with $69.80 \%$ and $72.76 \%$ respectively, whereas treatment $\mathrm{C} 3$ had the lowest biological efficiency (51.72\%). Loss of organic material reached $50.56 \%$ for $\mathrm{C} 1 ; 44.50 \%$ for $\mathrm{C} 2 ; 40.16 \%$ for $\mathrm{C} 3$. Nutrition analysis of mushrooms did not reveal any significant difference among treatments, except crude fiber, where treatment $\mathrm{C} 3$ was higher by $18.95 \%$, followed by treatment C2 with $16.40 \%$ and control with $14.95 \%$.
\end{abstract}

KEY WORDS: Nutrition analysis; Fungi; Productivity; Residues.

\section{INTRODUÇÃO}

O cultivo de cogumelos, como o Pleurotus ostreatus, é realizado por meio da utilização de compostos à base de resíduos lignocelulósicos. O cogumelo $P$. ostreatus faz parte dos chamados fungos de decomposição branca, que possuem suporte enzimático altamente especializado, capaz de degradar componentes da madeira como lignina, celulose e hemicelulose (ESPOSITO et al., 2010).

Muitos tipos de compostos já foram avaliados, sendo que a palha da cana-de-açúcar é uma das principais matérias-primas utilizadas para o cultivo de cogumelos no Estado de São Paulo. No entanto, poucos trabalhos têm sido feitos para testar 
a eficiência da palha de sorgo no cultivo de cogumelos comestíveis.

Segundo May et al. (2013), existem diferentes tipos de sorgo: o sorgo granífero, usado na alimentação animal, o sorgo biomassa, utilizado para produção de matéria-prima lignocelulósica, e o sorgo sacarino, utilizado para a produção de etanol. A grande variabilidade desta cultura a torna capaz de atingir os mais diferentes mercados.

No Brasil o sorgo sacarino tem sido utilizado como uma cultura complementar a cana-de-açúcar, visando o fornecimento de matéria-prima nos períodos de entressafra (MAY et al., 2013). Outra característica vantajosa do cultivo de sorgo é sua resistência ao estresse hídrico, que mesmo sendo uma planta xerófita apresenta eficientes mecanismos de tolerância à seca, com variedades adaptadas a diferentes zonas climáticas (LANDAU; SANS, 2010).

O sorgo pertence à família Poacea (= Gramineae), e por ser uma planta C4, produz alta quantidade de biomassa, com elevada relação C/N. Tamanini (2004) descreve a palha de sorgo como sendo composta por 34\% de celulose, $44 \%$ de hemicelulose e $24 \%$ de lignina. Ao avaliar a composição bromatológica de oito híbridos de sorgo, Pedreira et al. (2003)-constataram na matéria seca entre 34,2 a 25,7\% de celulose, 27,41 a 20,12\% de hemicelulose e 4,47 a 12,61\% de lignina. Essas e outras características da planta de sorgo mostram-se como uma alternativa promissora em relação à cana-de-açúcar, para a produção de cogumelos comestíveis no Estado de São Paulo.

O objetivo deste estudo foi analisar a viabilidade de uso da palha de sorgo sacarino e sorgo biomassa na formulação de novos compostos para o cultivo do $P$. ostreatus, levando-se como critérios de avaliação a caracterização química dos substratos, a perda de matéria orgânica, a eficiência biológica, bem como a caracterização nutricional dos cogumelos.

\section{MATERIAL E METODOS}

O experimento foi feito em duas etapas: 1. Compostagem e pasteurização, conduzido na Faculdade de Ciências Agronômicas (FCA/UNESP), Botucatu (SP), uti- 
lizando o pátio de compostagem e pasteurização; 2. Incubação e colheita, conduzido na Universidade do Sagrado Coração (USC), em estufa experimental 4 x 6 m, instalada nas dependências da instituição.

Foram testadas duas formulações de compostos, à base de sorgo biomassa (C2) e sorgo sacarino (C3), sendo que a coleta destas palhas foi realizada na fazenda experimental USC, os quais foram comparados com um composto tradicional (testemunha) à base de palha de cana-de-açúcar (C1) (Tabela 1).

Tabela 1. Formulação dos tratamentos.

\begin{tabular}{|c|c|c|c|c|c|c|c|c|}
\hline & & $\begin{array}{c}\text { Umi } \\
\text { (\%) }\end{array}$ & $\begin{array}{c}\text { Peso } \\
\text { Úmido } \\
\text { (kg) }\end{array}$ & $\begin{array}{l}\text { Peso } \\
\text { Seco } \\
(\mathrm{kg})\end{array}$ & C (\%) & $\mathrm{C}(\mathrm{Kg})$ & $\mathbf{N}(\%)$ & $\mathrm{N}(\mathrm{kg})$ \\
\hline \multirow{3}{*}{$\mathrm{C} 1$} & Palha de cana & 5 & 101,56 & 96,48 & 48 & 46,31 & 0,60 & 0,58 \\
\hline & Farelo de trigo & 6 & 6,50 & 6,11 & 46 & 2,8 & 2,50 & 0,15 \\
\hline & TOTAL & & 108,06 & 102,59 & & 49,12 & & 0,73 \\
\hline \multirow{3}{*}{$\mathrm{C} 2$} & Sorgo biomassa & 5 & 67,83 & 64,44 & 48 & 30,93 & 0,70 & 0,70 \\
\hline & Farelo de trigo & 6 & 0,80 & 0,75 & 46 & 0,35 & 2,50 & 0,02 \\
\hline & TOTAL & & 68,63 & 65,19 & & 31,28 & & 0,72 \\
\hline \multirow{3}{*}{ C3 } & Sorgo sacarino & 5 & 65,72 & 62,43 & 48,00 & 29,97 & 0,40 & 0,25 \\
\hline & Farelo de trigo & 6 & 11,50 & 10,81 & 46,00 & 4,97 & 2,50 & 0,27 \\
\hline & TOTAL & & 77,22 & 73,24 & & 34,94 & & 0,52 \\
\hline
\end{tabular}

Nota: Umi $=$ Umidade; $\mathrm{C}=$ Carbono; $\mathrm{N}=$ Nitrogênio.

A colheita do sorgo biomassa foi realizada através de colheita mecânica, com o uso do Trator Massey Ferguson 275 4x4 e Ensiladeira Tarup, o sorgo sacarino foi colhido manualmente, ambas as plantas foram empregadas inteiras, secadas e moídas para a retirada da sacarose em uma moenda de cana elétrica, logo após esse processo foi realizada a trituração do material em uma Ensiladeira Tarup.

A Pré-Compostagem e Fase I de compostagem foram realizadas em barracão aberto, com cobertura em chapa galvanizada e piso de concreto. Dois dias antes de formar as leiras, as palhas de sorgo (sacarino e biomassa) e cana-de-açúcar foram 
umedecidas com água corrente utilizando mangueira para 75\% de umidade média (Pré-umedecimento). Posteriormente iniciou-se a Fase I da compostagem, quando as leiras foram formadas por uma camada de palha já umedecida $(20 \mathrm{~cm}$ de altura), seguido da adição de farelo de trigo, gesso e calcário, conforme cada tratamento (Tabela 1). As leiras foram reviradas com 2 e 4 dias, até alcançarem 1,8 m x 1,8 m de altura e largura respectivamente. Todos os compostos foram ajustados para uma relação C/N de 67/1.

$\mathrm{Na}$ fase II, os compostos foram transferidos para caixas treliçadas sendo posteriormente dispostos aleatoriamente dentro de uma câmara climatizada (Dalsem Mushrooms) para a pasteurização no sexto dia (8 horas a $\left.62^{\circ} \mathrm{C} \pm 2^{\circ} \mathrm{C}\right)$ e condicionamento no sétimo dia $\left(4\right.$ dias a $\left.48^{\circ} \mathrm{C} \pm 2^{\circ} \mathrm{C}\right)$.

No final das fases I e II da compostagem, três amostras de cada leira foram recolhidas e desidratadas a $65^{\circ} \mathrm{C}$ durante 48 horas para análise de carbono, nitrogênio, matéria orgânica e $\mathrm{pH}$. Este mesmo procedimento foi repetido ao final do ciclo de cultivo. Estas análises foram feitas no Laboratório de Análise Química de Fertilizantes e Corretivos, do Departamento de Recursos Naturais - Ciência do Solo - FCA/ UNESP, Botucatu (SP), de acordo com a metodologia descrita em EMBRAPA (2013).

No $11^{\circ}$ dia, foi realizada a inoculação do composto com a linhagem de $P$. ostreatus POS-13/114, adquirida junto à Micoteca do Módulo de Cogumelos, FCA/ UNESP, Botucatu-(SP). O preparo do inóculo foi feito por meio da metodologia proposta por Minhoni et al. (2005). A inoculação do composto foi realizada de forma manual, no interior da câmara climatizada Dalsem, com higienização dos utensílios utilizados (bandeja, tesoura e copo dosador de inóculo) com álcool 70\%. A proporção de inóculo utilizada foi de $40 \mathrm{gkg}^{-1}$ de massa fresca do composto.

A incubação foi feita na estufa experimental da USC, durante 30 dias, condicionada a uma temperatura média de $25{ }^{\circ} \mathrm{C}$ e umidade relativa de 55-70\%. A colheita foi realizada durante 60 dias, sendo anotados os pesos de basidiomas de cada pacote.

As análises nutricionais dos cogumelos foram realizadas no Laboratório de Bromatologia da Faculdade de Medicina Veterinária e Zootecnia - FMVZ/UNESP, Botucatu (SP). Foram analisadas três amostras de basidiomas inteiros, desidratados e moídos, por tratamento. No total foram analisadas nove amostras de cogumelos (três por tratamento), sendo avaliados os seus teores de proteína bruta, extrato eté- 
reo, cinzas e fibra bruta, de acordo com a metodologia de Silva e Queiroz (2002).

A perda de matéria orgânica (PMO) representa o índice utilizado para quantificar decomposição do substrato pelo fungo sofrido durante o cultivo e foi realizada segundo Rajarathnam e Bano (1989). Tal índice foi baseado na perda da matéria orgânica decomposta pelo fungo que foi determinada por meio da diferença entre a massa seca do substrato inicial, e a massa seca do substrato residual (póscolheita). Assim, a PMO foi avaliada por meio da seguinte fórmula:

A produtividade foi expressa também por meio da eficiência biológica (EB), que consiste na quantificação da biomassa de substrato, convertida em biomassa fúngica.

Os dados obtidos foram submetidos à análise de variância e as médias comparadas pelo teste de comparações múltiplas de Tukey com 5\% de significância (SNEDECOR; COCHRAN, 1972). Para facilitação da precisão dos cálculos, foi utilizado o software SISVAR 4.2, desenvolvido pelo Departamento de Ciências Exatas da Universidade Federal de Lavras (MG) (UFLA).

\section{RESULTADOS E DISCUSSÃO}

Os resultados da análise química dos compostos no final das fases I e II da compostagem e do final do ciclo de cultivo (composto exaurido) podem ser visualizados nas Tabelas 2,3 e 4 .

Tabela 2. Análise química do composto do final da Fase I da compostagem.

\begin{tabular}{lllllll}
\hline Tratamento & $\mathbf{N}(\%)$ & $\mathbf{M O}(\%)$ & $\mathbf{C}(\%)$ & $\mathbf{C} / \mathbf{N}$ & Umidade & $\mathbf{p H}$ \\
\hline C1 & $0,15^{\mathrm{a}}$ & $20,0^{\mathrm{a}}$ & $11,0^{\mathrm{a}}$ & $80,0^{\mathrm{a}}$ & $73,0^{\mathrm{b}}$ & $7,7^{\mathrm{a}}$ \\
$\mathrm{C} 2$ & $0,2^{\mathrm{a}}$ & $19,0^{\mathrm{a}}$ & $11,0^{\mathrm{a}}$ & $55,0^{\mathrm{a}}$ & $77,0^{\mathrm{a}}$ & $8,0^{\mathrm{a}}$ \\
$\mathrm{C} 3$ & $0,2^{\mathrm{a}}$ & $19,0^{\mathrm{a}}$ & $11,0^{\mathrm{a}}$ & $55,0^{\mathrm{a}}$ & $78,0^{\mathrm{a}}$ & $8,1^{\mathrm{a}}$ \\
CV (\%) & 22,27 & 8,45 & 7,42 & 25,78 & 1,07 & 0,00 \\
DMS & 0,17 & 6,82 & 3,41 & 68,22 & 3,41 & 1,27 \\
\hline
\end{tabular}

Nota: $\mathrm{C} 1$ = Composto à base de cana-de-açúcar (testemunha); $\mathrm{C} 2=$ Composto à base de sorgo biomassa; $\mathrm{C} 3=$ Composto à base de sorgo sacarino; $\mathrm{CV}=$ Coeficiente de variação; $\mathrm{DMS}=$ Diferença mínima significativa; $\mathrm{N}=$ nitrogênio; $\mathrm{MO}=$ Matéria orgânica; $\mathrm{C}=$ Carbono; $\mathrm{C} / \mathrm{N}=$ Relação carbono/nitrogênio; $\mathrm{pH}=$ Potencial hidrogeniônico $=-\log \left[\mathrm{H}^{+}\right]$. Médias seguidas por letras minúsculas iguais na coluna não diferenciam estatisticamente entre si (Tukey, 5\%). 
Tabela 3. Análise química do composto do final da Fase II da compostagem.

\begin{tabular}{lcccccc}
\hline Tratamento & $\mathrm{N}(\%)$ & $\mathrm{MO}(\%)$ & $\mathrm{C}(\%)$ & $\mathrm{C} / \mathrm{N}$ & Umidade & $\mathrm{pH}$ \\
\hline $\mathrm{C} 1$ & $0,2^{\mathrm{a}}$ & $24,5^{\mathrm{a}}$ & $13,5^{\mathrm{a}}$ & $67,5^{\mathrm{a}}$ & $69,0^{\mathrm{b}}$ & $7,7^{\mathrm{b}}$ \\
$\mathrm{C} 2$ & $0,2^{\mathrm{a}}$ & $20,0^{\mathrm{a}}$ & $11,0^{\mathrm{a}}$ & $55,0^{\mathrm{a}}$ & $77,0^{\mathrm{a}}$ & $8,15^{\mathrm{a}}$ \\
$\mathrm{C} 3$ & $0,2^{\mathrm{a}}$ & $22,5^{\mathrm{a}}$ & $12,5^{\mathrm{a}}$ & $62,5^{\mathrm{a}}$ & $73,5^{\mathrm{ab}}$ & $8,20^{\mathrm{a}}$ \\
$\mathrm{CV}(\%)$ & 0,00 & 9,32 & 10,47 & 10,47 & 2,30 & 0,51 \\
DMS & 0,00 & 8,69 & 5,39 & 26,96 & 7,0 & 0,17 \\
\hline
\end{tabular}

Nota: C1 = Composto à base de cana-de-açúcar (testemunha); C2 = Composto à base de sorgo biomassa; $\mathrm{C} 3$ = Composto à base de sorgo sacarino; $\mathrm{CV}=$ Coeficiente de variação; DMS = Diferença mínima significativa; $\mathrm{N}=$ nitrogênio; $\mathrm{MO}=$ Matéria orgânica; $\mathrm{C}=$ Carbono; $\mathrm{C} / \mathrm{N}=$ Relação carbono/nitrogênio; $\mathrm{pH}=$ Potencial hidrogeniônico $=-\log \left[\mathrm{H}^{+}\right]$. Médias seguidas por letras minúsculas iguais na coluna não diferenciam estatisticamente entre si (Tukey, 5\%).

Tabela 4. Análise química do composto do final do ciclo de cultivo (composto exaurido).

\begin{tabular}{lcccccc}
\hline Tratamento & $\mathrm{N}(\%)$ & $\mathrm{MO}(\%)$ & $\mathrm{C}(\%)$ & $\mathrm{C} / \mathrm{N}$ & Umidade & $\mathrm{pH}$ \\
\hline C1 & $0,25^{\mathrm{a}}$ & $18,0^{\mathrm{a}}$ & $10,0^{\mathrm{a}}$ & $41,0^{\mathrm{a}}$ & $69,5^{\mathrm{b}}$ & $5,5^{\mathrm{a}}$ \\
$\mathrm{C} 2$ & $0,20^{\mathrm{a}}$ & $18,0^{\mathrm{a}}$ & $10,0^{\mathrm{a}}$ & $50,0^{\mathrm{a}}$ & $76,5^{\mathrm{a}}$ & $5,5^{\mathrm{a}}$ \\
$\mathrm{C} 3$ & $0,20^{\mathrm{a}}$ & $17,0^{\mathrm{a}}$ & $9,5^{\mathrm{a}}$ & $47,5^{\mathrm{a}}$ & $77,5^{\mathrm{a}}$ & $5,3^{\mathrm{a}}$ \\
$\mathrm{CV}(\%)$ & 18,84 & 8,00 & 12,46 & 12,16 & 0,95 & 3,43 \\
DMS & 0,17 & 5,90 & 5,11 & 23,44 & 2,95 & 0,78 \\
\hline
\end{tabular}

Nota: C1 = Composto à base de cana-de-açúcar (testemunha); C2 = Composto à base de sorgo biomassa; $\mathrm{C} 3=$ Composto à base de sorgo sacarino; $\mathrm{CV}=$ Coeficiente de variação; $\mathrm{DMS}=$ Diferença mínima significativa; $\mathrm{N}=$ nitrogênio; $\mathrm{MO}=$ Matéria orgânica; $\mathrm{C}=$ Carbono; $\mathrm{C} / \mathrm{N}=$ Relação carbono/nitrogênio; $\mathrm{pH}=$ Potencial hidrogeniônico $=-\log \left[\mathrm{H}^{+}\right]$. Médias seguidas por letras minúsculas iguais na coluna não diferenciam estatisticamente entre si (Tukey, 5\%).

Não houve diferença significativa entre os tratamentos nas Fases I e II da compostagem, com exceção da umidade, da qual o tratamento C1 (Testemunha) apresentou um valor significativamente inferior aos tratamentos a $\mathrm{C} 2$ e $\mathrm{C} 3$, com valor 73,0, 77,0 e 78,0\% respectivamente, no final da Fase I (Tabelas 2 e 3). No final da fase II o tratamento C1 também apresentou resultados de umidade significativamente inferiores aos compostos à base de sorgo (69\%), assim como no final do experimento onde a umidade do tratamento $\mathrm{C} 1$ foi de $69,5 \%$, logo abaixo dos tratamentos C2 e C3 que não diferiram significativamente entre si, com valores de 76,5 e $77,5 \%$, respectivamente (Tabela 3). Possivelmente os resultados do teor de umidade podem ter sido influenciados pela granulometria dos compostos, que foi visivelmen- 
te menor para os compostos à base de sorgo biomassa e sacarino se comparado ao composto à base de palha de cana-de-açúcar, resultando em uma maior densidade $\mathrm{e}$ compactação, já que estes materiais foram adquiridos de formas diferentes e colhidos com máquinas diferentes.

Esse fenômeno pode ser explicado pelas propriedades físico-químicas da água, que resulta em seu comportamento em um meio particulado. Nesse meio, a água é retida pela superfície sólida por meio de forças de adesão e coesão, resultando em um fenômeno chamado de capilaridade. Neste fenômeno, ocorre a elevação de uma coluna de água, quando um tubo de pequeno diâmetro é inserido em um reservatório com água. A altura da coluna de água é inversamente proporcional ao diâmetro do tubo (NETTO, 2010).

Em um material granulado existe uma porção de sólidos e uma porção porosa, esta porção porosa é parte preenchida por ar e parte preenchida por água. Quanto menor o tamanho dos grânulos, maior a área superficial da parte sólida, consequentemente maior retenção de água, por meio dos fenômenos já mencionados. De uma forma simplificada, o volume dos grânulos é diretamente proporcional ao volume de poros, e quanto maior os poros maior a quantidade de ar, e quanto menor os poros maior a quantidade de água (LIER, 2010).

Houve diferença significativa de produtividade, mensurada pela massa de basidiomas frescos (MBF) entre os tratamentos C1, C2 e C3, com 1730,0, 1340,0 e $1070,0 \mathrm{~g}$, respectivamente (Tabela 5). Os tratamentos C1 e C2 não diferiram significativamente entre si para eficiência biológica (EB), com valores de 69,80 e 72,76\%, foram superiores ao tratamento C3 (51,72\%). Houve diferença significativa entre os tratamentos para a perda de matéria orgânica (PMO), sendo que o C1 foi superior com PMO de 50,56\% e logo abaixo os tratamentos C2 e C3 com 44,50 e 40,16\%, respectivamente.

A granulometria e densidade dos compostos pode ter influenciado também a produtividade expressa em massa de basidiomas frescos, a eficiência biológica e perda de matéria orgânica, já que quimicamente os tratamentos não diferiram significativamente (Tabelas 2 e 3). Oliveira (2000) avaliou a eficiência biológica (EB) de duas linhagens de $P$. ostreatus em diferentes granulometrias e obteve $\mathrm{EB}$ de 8,45 e $19,40 \%$ em bagaço de cana picado, e uma eficiência biológica quase nula $(0,84 \%)$ 
para bagaço de cana em pó. Este mesmo autor atribuiu esses resultados ao excesso de nitrogênio e também a pequena granulometria, que causa a compactação, e dificulta as trocas gasosas realizadas pelo fungo na respiração. Paz et al. (2013) testaram a eficiência de Pleurotus sajor-caju, em bagaço de cajá por meio de dois tratamentos $50 \%$ bagaço de cana, $50 \%$ bagaço de cajá, e $25 \%$ cana $75 \%$ bagaço de cajá, e foi obtida eficiência biológica de 28,94 e 26,37\% respectivamente, e esses resultados foram atribuídos à obstrução dos poros dos compostos, devido à liberação de pectina proveniente do bagaço de cajá nesses poros, o que dificultou as trocas gasosas e diminuiu a eficiência biológica.

Tabela 5. Os resultados de produtividade estão expressos como massa de basidiomas fres$\cos (\mathrm{MBF})$, eficiência biológica (EB) e perda de matéria orgânica (PMO).

\begin{tabular}{lccc}
\hline Tratamento & MBF $(\mathrm{g})$ & EB $(\%)$ & PMO (\%) \\
\hline C1 & $1730,0^{\mathrm{a}}$ & $69,80^{\mathrm{a}}$ & $50,56^{\mathrm{a}}$ \\
$\mathrm{C} 2$ & $1340,0^{\mathrm{b}}$ & $72,76^{\mathrm{a}}$ & $44,50^{\mathrm{b}}$ \\
C3 & $1070,0^{\mathrm{c}}$ & $51,72^{\mathrm{b}}$ & $40,16^{\mathrm{c}}$ \\
CV (\%) & 23,89 & 23,51 & 10,99 \\
DMS & 0,25 & 11,58 & 3,76 \\
\hline
\end{tabular}

Nota: $\mathrm{C} 1=$ Composto à base de cana-de-açúcar (testemunha); $\mathrm{C} 2=$ Composto à base de sorgo biomassa; $\mathrm{C} 3$ = Composto à base de sorgo sacarino; $\mathrm{CV}=$ Coeficiente de variação; DMS = Diferença mínima significativa. Médias seguidas por letras minúsculas iguais na coluna não diferenciam estatisticamente entre si (Tukey, 5\%).

Os resultados da análise nutricional dos basidiomas podem ser visualizados na Tabela 6, onde pode ser verificado que não houve diferença significativa entre os tratamentos para proteína bruta. Estes resultados estão de acordo com os teores de proteína bruta encontrados em P. ostreatus descritos por Chang e Miles (2004), onde tais teores variaram entre 10,5 a $30,4 \%$. 
Tabela 6. Análise nutricional do cogumelo Pleurotus ostreatus em função do tipo de composto.

\begin{tabular}{lccccc}
\hline Tratamento & MS (\%) & PB (\%) & EE (\%) & FB (\%) & MM (\%) \\
\hline C1 & $85,4^{\mathrm{a}}$ & $16,05^{\mathrm{a}}$ & $1,70^{\mathrm{a}}$ & $14,95 \mathrm{~b}$ & $8,00^{\mathrm{a}}$ \\
$\mathrm{C} 2$ & $86,15^{\mathrm{a}}$ & $15,35^{\mathrm{a}}$ & $1,35^{\mathrm{a}}$ & $16,40^{\mathrm{ab}}$ & $8,85^{\mathrm{a}}$ \\
$\mathrm{C} 3$ & $86,70^{\mathrm{a}}$ & $16,30^{\mathrm{a}}$ & $1,55^{\mathrm{a}}$ & $18,95^{\mathrm{a}}$ & $8,40^{\mathrm{a}}$ \\
CV (\%) & 0,94 & 3,10 & 11,30 & 5,67 & 3,91 \\
DMS & 3,36 & 2,06 & 0,72 & 3,97 & 1,37 \\
\hline
\end{tabular}

Nota: $\mathrm{C} 1$ = Composto à base de cana-de-açúcar (testemunha); $\mathrm{C} 2=$ Composto à base de sorgo biomassa; $\mathrm{C} 3=$ Composto à base de sorgo sacarino; $\mathrm{CV}=$ Coeficiente de variação; $\mathrm{DMS}=$ Diferença mínima significativa. $\mathrm{MS}=$ Massa seca; $\mathrm{PB}=$ Proteína bruta; $\mathrm{EE}=$ Extrato etéreo; $\mathrm{FB}=$ Fibra bruta; $\mathrm{MM}=$ Material mineral. Médias seguidas por letras minúsculas iguais na coluna não diferenciam estatisticamente entre si (Tukey, 5\%).

Os cogumelos comestíveis são alimentos popularmente procurados por suas propriedades nutricionais e seu sabor, além de possuírem alto teor de proteínas e baixo teor de gorduras, fato que pode ser confirmado pelos valores obtidos para o teor de lipídios (extrato etéreo), que não variaram significativamente entre os tratamentos C1, C2 e C3, com valores de 1,70, 1,35 e 1,55, respectivamente (Tabela 6). Sturion e Oetterer (1995), ao avaliarem a composição nutricional de $P$. ostreatus em diferentes substratos, obtiveram um teor de lipídios de 1,54 e 1,86\%, valores semelhantes aos obtidos no presente experimento.

A fibra bruta refere-se aos carboidratos não digestíveis ao homem e outros animais monogástricos. Os valores de fibra bruta (FB) diferiram significativamente entre si, onde o tratamento $\mathrm{C} 3$ apresentou resultado superior a C2 e C1, com 18,95, 16,40 e $14,95 \%$, respectivamente (Tabela 6). Os valores de fibra bruta podem variar em função do substrato utilizado para cultivo, conforme o que foi demonstrado por Silva, Costa e Clemente (2002), onde foi avaliada a composição nutricional de Pleurotus pulmonaris em três diferentes tipos de substrato (resíduo de algodão, capim Cymbopogon citratus, capim Panicum maximum) e foi verificado que o resíduo de algodão apresentou maiores valores de fibra bruta, com 9, 4 e 4,94\% respectivamente. Silva, Dias e Siqueira (2007), ao realizarem um experimento com o cogumelo Pleurotus sajor-caju, constataram um aumento no teor de fibra bruta em função do aumento na concentração de nitrogênio presente na composição do substrato. 
O que não foi observado neste experimento, já que o teor de nitrogênio e a relação $\mathrm{C} / \mathrm{N}$ dos três compostos não diferiram significativamente.

\section{CONCLUSÃO}

Os compostos à base de palha de sorgo biomassa e sorgo sacarino demonstraram valores de produtividade inferiores ao composto testemunha (à base de cana-de-açúcar). Entretanto, a eficiência biológica do composto à base de sorgo biomassa não diferiu significativamente da testemunha, demonstrando que esse pode ainda ser uma alternativa promissora para a produção de $P$. ostreatus.

A maior compactação dos pacotes dos tratamentos com sorgo pode ter influenciado os resultados da pesquisa, tornando-se assim necessárias novas experimentações com diferentes compactações.

\section{AGRADECIMENTOS}

À Fundação de Amparo à Pesquisa do Estado de São Paulo (FAPESP) pela concessão de bolsa de iniciação científica do primeiro autor (PROCESSO 2015/16400-3).

\section{REFERÊNCIAS}

CHANG, S. T.; MILES, P. G. Mushrooms: cultivation, nutritional value, medicinal effect, and environmental impact. 2. ed. Boca Raton: CRC, 2004. 480 p.

EMBRAPA. Sistema brasileiro de classificação de solos. Rio de Janeiro: Centro Nacional de Pesquisa de Solos, 2013.

ESPOSITO, E. et al. Fungos: uma introdução a biologia bioquímica e biotecnologia. Caxias do Sul: Educs, 2010.

LANDAU, E. C.; SANS, L. M. A. Cultivo de sorgo. Sete Lagoas, Embrapa Milho Sor- 
go, 2010. Disponível em: < http://www.cnpms.embrapa.br/publicacoes/sorgo_6_ ed/clima.htm > Acesso em: 05 mar. 2015.

LIER, Q. J. V. Física do Solo. Viçosa: UFV, 2010.

MAY, A. et al. Cultivo de sorgo sacarino em área de reforma de canavial. Sete Lagoas: Embrapa Milho Sorgo, 2013. (Circular Técnica, 186).

MINHONI, M. T. A. et al. Cultivo de Lentinula edodes (Berk.) Pegler - (Shiitake). 2. ed. Botucatu: FEPAF, 2005.

NETTO, A. Manual de Hidráulica. São Paulo: Blucher, 2010.

OLIVEIRA, H. C. B. Avaliação de três substratos com diferentes granulometrias, para o cultivo de duas linhagens de Pleurotus ostreatus (Jacq.:Fr.) Kummer. 2000. 89f. Dissertação - Universidade Federal do Ceará, Fortaleza, 2000.

PAZ, M. F. et al. Cultivation of edible mushroom Hiboukitake in caja bagasse by in Jun-Cao technique. Journal of Biotechnology and Biodiversity, Gurupi, v. 4, n. 2, p. 146-152, 2013.

PEDREIRA, M. S. et al. Características agronômicas e composição química de oito híbridos de sorgo. Revista Brasileira de Zootecnia, Jaboticabal, v. 32, n. 5, p. 1083-1092, 2003.

RAJARATHNAM, S.; BANO, Z. Pleurotus Mushrooms; part 3: Biotransformation of natural lignocellulosic waste: commercial applications and implications. Critical Reviews in Food Science and Nutrition, Boca Raton, v. 28, n. 1, p. 31-113, 1989.

SILVA, S. O.; COSTA, S. M. G.; CLEMENTE, E. Chemical composition of Pleurotus pulmonarius (Fr.) Quél., substrates and residue after cultivation. Braz. arch. biol. technol. Curitiba, v. 45, n. 4, 2002.

SILVA, E. G.; DIAS, E. S.; SIQUEIRA, F. G. Análise química de corpos de frutificação de Pleurotus sajor-caju cultivado em diferentes concentrações de nitrogênio. Ciênc. Tecnol. Aliment., Campinas, v. 27, n. 1, p. 72-75, 2007. 
SILVA, D. J.; QUEIROZ, A. C. Análise de alimentos: métodos químicos e biológicos. Viçosa: UFV, 2002.

SNEDECOR, G. W. E.; COCHRAN, W. G. Statistical methods. $6^{\text {th }}$ ed. Ames: Iwoa State University Press, 1972.

STURION, G. L.; OETTERER, M. Composição química de cogumelos comestíveis (Pleurotus spp.) originados de cultivos em diferentes substratos. Ciência e Tecnologia de Alimentos, Campinas, v. 15, n. 2, 1995.

TAMANINI, C.; HAULY, M. C. O. Resíduos agroindustriais para produção biotecnológica de xilitol. Ciências de Alimentos. Londrina, v. 25, n. 4, p. 315-330, 2004.

Recebido em: 27/06/2017

Aceito em: 24/05/2018 